\title{
Die Ideologien beim Wort nehmen: Soziale Verantwortung von Unternehmen inhaltlich und institutionell sichern!*
}

\begin{abstract}
1 Vorbemerkung
Seit einigen Jahren steht mit der Nachhaltigen Unternehmensführung ein auf ein ganzheitlich tragfähiges Wirtschafts- und Lebensmodell bezogenes Wirtschaften im Fokus meines wissenschaftlichen Bemühens. Nachhaltige Unternehmensführung wird aber gerade in jüngster Zeit insbesondere in der Unternehmenspraxis immer häufiger als Corporate (Social) Responsibility (C(S)R) buchstabiert und damit auf die gesellschaftliche Verantwortung der Unternehmen bzw. Unternehmer zurückverwiesen, aus der heraus sie vermeintlich entspringt und in deren Umsetzung sie sich praktisch offenbart.

Hier setzen die Gedanken an, die ich in diesem Beitrag vortragen möchte. Denn ich halte das CSR-Konzept, insbesondere in seiner in der Praxis vorherrschenden Ausprägung für hochgradig ideologieverdächtig und empfehle Praktikern und Theoretikern dringend die bis heute erfrischende Lektüre des einschlägigen Aufsatzes von Horst Steinmann aus dem Jahre 1973, an den ich meine nachfolgenden Ausführungen anknüpfe. Das scheint mir berechtigt, ja geradezu notwendig, denn ich finde ihn zwar hier und da in den Literaturverzeichnissen aktueller Veröffentlichungen zum Thema aufgeführt, aber seine Thesen nicht wirklich rezi-

\footnotetext{
* Erstveröffentlichung in: Baumann, W./Braukmann, U./Mathes, W. (Hrsg.): Innovation und Internationalisierung, Festschrift für Norbert Koubek, Wiesbaden 2010, 129-149.
} 
piert und gewürdigt. Das mag daran liegen, dass er „uralt“ ist und zudem in Deutsch verfasst und daher der internationalen Diskussion nicht zugänglich. Deshalb will ich zumindest die inhaltliche Lücke schließen und anknüpfend daran versuchen, eine Fortschreibung aus heutiger Sicht vorzunehmen, indem ich die Kritik konstruktiv wende und Gestaltungsmöglichkeiten der glaubwürdigen Umsetzung sozialer Verantwortungsübernahme von Unternehmen diskutiere.

\section{Was meint CSR heute?}

Die jüngere Diskussion um CSR wurde im angelsächsischen Sprachraum entfacht. Hintergrund der Debatte dort waren die gehäuft zutage getretenen Unternehmensskandale, die sich zumindest teilweise sogar in explizit gesetzeswidrigen Handlungen von Managern offenbarten. Hintergrund war und ist zudem die in den USA weit mehr als z.B. in Deutschland verbreitete Gewohnheit von Unternehme(r)n, in größerem Umfang als Spendengeber für kulturelle und soziale Projekte in Erscheinung zu treten. Dementsprechend treten die Aspekte der good corporate governance sowie des philanthropischen Engagements deutlich in den Vordergrund und prägen die Debatte. ${ }^{1}$

Das drückt sich z.B. in der viel zitierten Carroll-Pyramide aus, die CSR auf vier Pyramidenstufen verortet, deren unterste die ökonomische Verantwortung (,,be profitable“) ausmacht, der auf der zweiten Ebene die Einhaltung der einschlägigen Gesetze als Ausdruck gesetzlicher Verantwortung folgt (,obey the law“). Darüber ist die ethische Verantwortung im Sinne einer Beachtung von Fairness und Gerechtigkeit angesiedelt (,be ethical“), gefolgt vom bürgerschaftlichen Engagement als Förderer sozialer Anliegen (,be a good corporate citizen“) (Carroll 1991).

Eine andere Akzentuierung zeigt sich in den europäischen Auseinandersetzungen mit CSR. So wird in der quasi-offiziellen Begriffsdefinition der EU Kommission CSR verstanden als

„ein Konzept, das den Unternehmen als Grundlage dient, auf freiwilliger Basis soziale Belange und Umweltbelange in ihre Unternehmenstätigkeit und in die Wechselbeziehungen mit den Stakeholdern zu integrieren. Sozial verantwortlich handeln heißt nicht nur, die gesetzlichen

${ }^{1}$ Vgl. hierzu z.B. Matten/Moon 2008, Habisch/Wegener 2005 
Bestimmungen einhalten, sondern über die bloße Gesetzeskonformität hinaus ,mehr' investieren in Humankapital, in die Umwelt und in die Beziehungen zu anderen Stakeholdern (EU-Kommission 2001, 7).

Zentral an dieser Definition sind zum einen die Freiwilligkeit, zum anderen der Einbezug in die Unternehmenstätigkeit und in die darüber hinaus bestehenden Stakeholderbeziehungen etwa in Form von Sponsoring oder Volunteering. Zentral ist darüber hinaus der Verweis darauf, worin das soziale Engagement sich substantiell äußern sollte, nämlich über die Einhaltung aller einschlägigen gesetzlichen Bestimmungen hinaus in Investitionen in Humankapital und Umwelt sowie in der Beziehungspflege zu den Stakeholdern.

Damit beinhaltet auch dieses Begriffsverständnis das sog. Corporate Citizenship, ${ }^{2}$ aber eben nicht nur dieses, sondern eine insgesamt verantwortliche Ausübung der eigenen Geschäftstätigkeit. Mit dem inhaltlichen Bezug auf Investitionen in das Humankapital und auf Umweltbelange wird die Brücke zur Corporate Sustainability geschlagen, ${ }^{3}$ hier in der Variante des sog. Drei Säulen-Modells, worauf noch zu sprechen zu kommen ist.

Auffällig ist zunächst, dass beide Definitionen, insbesondere aber die von Carroll, so verstanden werden können, dass sie ein Handeln einschließen, das bei genauerem Hinsehen kaum als Ausdruck sozialer Verantwortungsübernahme angesehen werden kann: die Gewinnerzielung (bei Carroll) und die Einhaltung der gesetzlichen Vorschriften, die ein Unternehmen betreffen (in beiden Fällen).

Für Carroll sind und bleiben die Eigentümer damit die relevantesten Stakeholder, für die das Unternehmen aus sozialer Verantwortung heraus Gewinne zu erzielen hat, wobei Gewinnerzielung als Pyramidensockel auch als die Basis für die Übernahme aller weiteren Aspekte sozialer Verantwortung interpretiert werden kann. Nächst bedeutsamer Stakeholder ist der Gesetzgeber, demgegenüber das Unternehmen seine soziale Verantwortung durch Einhaltung der gesetzlichen Vorschriften beweist. Fairness und Gerechtigkeit können als Prinzipien des Umgangs mit allen weiteren Anspruchsgruppen, aber damit eben auch als Ausdruck einer

${ }^{2}$ Vgl. hierzu auch Schrader 2003 sowie die begriffliche Meta-Analyse von Garriga/Melé 2004.

3 Siehe hierzu Dyllick/Hockerts 2002 sowie Freimann 2006. 
good corporate governance verstanden werden. Worin sie sich äußern könnten, bleibt unbestimmt. Die höchste Stufe stellt die Hergabe von Spenden und anderen freiwilligen sozialen Leistungen dar: Unternehmer/n als Finanziers für wünschenswerte, anders nicht finanzierbare gesellschaftliche Aktivitäten.

Die EU-Definition wird inhaltlich konkreter: „Soziale Belange und Umweltbelange" sollen in die Unternehmenstätigkeit und die Beziehungen zu den Anspruchsgruppen ,integriert“ werden, wobei die sozialen Belange fortfolgend als ,,investieren in Humankapital“ konkretisiert werden, Umweltbelange jedoch keine weitere Konkretisierung erfahren.

Festzuhalten bleibt an dieser Stelle, dass beide Begriffsverständnisse Dimensionen einschließen, die man durchaus auch als Ausdruck ,normalen“ erwerbswirtschaftlichen Geschäftsgebarens begreifen könnte, ${ }^{4}$ vor allem aber auf die soziale Verantwortung von Unternehmen ,beyond compliance“ abstellen und darin sowohl die Geschäftstätigkeit selbst (also den Prozess der Gewinnentstehung) als auch den der Gewinnverwendung in Form von Spenden und dergleichen einbeziehen.

Ideologie oder Ausdruck echten Bemühens um eine Perspektivenerweiterung für die Unternehmenspolitik? Bevor eine kritische Auseinandersetzung mit dieser Frage erfolgen kann, soll zunächst ein Rückblick auf zwei historische Vorläuferdiskurse genommen werden.

\section{Zur Geschichte der Forderung nach Corporate Social Responsibility}

Die Entwicklung der Sozialwissenschaften wird zwar von den Anhängern des sog. kritischen Rationalismus als Ausdruck des kontinuierlichen Bemühens um die immer bessere Erklärung der Welt idealisiert. Dem unvoreingenommenen Beobachter offenbart sich jedoch immer wieder die Tatsache, dass viele sozialwissenschaftliche Themen, oft angestoßen durch aktuelle gesellschaftliche Problemlagen, wissenschaftlich aufge-griffen und bearbeitet werden, zumeist ohne dass dabei ein (vorläufig) gesicherter Stand des Wissens erreicht wird. Dann ebbt der Diskurs ab, bis hin zum (vorübergehenden) völligen Verschwinden. Nach

\footnotetext{
${ }^{4}$ Auch in der EU-Definition kann man das ,nicht nur - sondern auch“ in Bezug auf Gesetzestreue und drüber hinausgehendes soziales Engagement durchaus so verstehen, dass auch Gesetzestreue Bestandteil von CSR sei.
} 
einer gewissen Zeit, nicht selten wieder ausgelöst durch einschlägige praktische Ereignisse, greift eine neue Generation von Forschern das Thema erneut auf. Es entwickelt sich, zumeist mit anderen Leitbegriffen oder Akzentuierungen, ein neuer Diskurs, der ebenfalls, ohne zum Konsens geführt zu haben, das Schicksal seiner Vorläufer teilt, nach einiger Zeit nicht mehr weitergeführt zu werden.

Der Diskurs über die gesellschaftliche Verantwortung von Unternehme(r)n ist ein prägnantes Beispiel für genau dieses Phänomen. Mindestens drei Wellen sind hier zu beobachten, von der die jeweils spätere(n) die frühere(n) zudem nur sehr begrenzt zur Kenntnis genommen haben.

Die erste Welle liegt schon lange zurück. Sie hat die Entstehungsphase der kaufmännischen Gewerbe Handel und Bankwesen begleitet. Denn beiden Gewerben haftete, vor allem bedingt durch das von der christlichen Kirche aus dem Altertum übernommene Zinsverbot der Makel der Amoralität an, im (internationalen) Handel verstärkt durch dessen Nähe zu Übervorteilung und Raub. Galt der Geschäftsinhalt des Geld-gegenGeld-Verleihens und des Geld-für-Mehrgeld-Verausgabens als unmoralisch, so konnten die Bankiers und Händler ihre soziale ,licence to operate“, wie man das im CSR-Jargon heute ausdrücken würde, nur dadurch aufbauen und sichern, dass sie einerseits die soziale Nähe zum (,ehrwürdigen") Adel suchten und angesichts dessen notorischer Finanzknappheit oft auch fanden und andererseits einen Habitus entwickelten, der ihre Gutbürgerlichkeit und Redlichkeit äußerlich in besonderer Weise versinnbildlichte.

Die Kenntnis und Einübung der Rituale „ehrbarer Kaufmannschaft“ waren so bis in das 20. Jahrhundert hinein Kernbestandteile der Kaufmannsausbildung in den Handelshochschulen (vgl. Löffelholz 1935, 118 ff.). Der Geruch der Unehrbarkeit färbte sogar noch auf die Anfänge der betriebswirtschaftlichen Wissenschaft ab, der mit dem Argument, sie sei doch nur öde Profitlehre, der Zugang zu den altehrwürdigen Universitäten zunächst verwehrt wurde (Weyermann/Schönitz 1912).

Die zweite Welle des Diskurses um die gesellschaftliche Verantwortung von Unternehmen wurde in den 70er Jahren des vergangenen Jahrhunderts angestoßen durch die Kritik, mit der sich das Unternehmertum von Seiten der 68er Studentenbewegung konfrontiert sah. Wer als Kapitalist und Ausbeuter tituliert wird, sieht sich in der sozialen Defensive und dadurch veranlasst, die positiven Leistungen, die Unternehmen für die Gesellschaft erbringen, in besonderer Weise herauszustellen. 
Diese Welle ist im deutschsprachigen Raum insbesondere durch zwei Erscheinungen charakterisiert: zum einen durch das sog. Davoser Manifest, einen auf dem 3. Europäischen Management Symposium in Davos im Februar 1973 verabschiedeten Kodex des ethischen Wohlverhaltens für die Unternehmensführung, zum anderen durch seit 1972 gehäuft von Unternehmen publizierte Sozialbilanzen, in denen in komprimierter Form der soziale Nutzen, den das bilanzierende Unternehmen stiftet, dargestellt und erläutert wird.

Das Davoser Manifest bezieht sich vor allem auf das Management von Großunternehmen, das es auffordert, verschiedenen Anspruchsgruppen sowie der Gesellschaft als Ganzes und sogar zukünftigen Generationen ,zu dienen und deren widerstreitende Interessen zum Ausgleich zu bringen, “ wozu ,,ausreichende Unternehmensgewinne erforderlich“ seien, die als „notwendiges Mittel, nicht aber Endziel der Unternehmensführung“ zu betrachten seien (zit. nach Steinmann 1973, 472-473).

Am Davoser Manifest setzt die Auseinandersetzung von Steinmann an, ohne dass es ihr jedoch meines Wissens gelungen wäre, hierüber im deutschsprachigen Schrifttum eine breitere Debatte anzustoßen. ${ }^{5}$ Seine kritischen Fragen:

- Wie verträgt sich die Auffassung von einer sozialen Unternehmensverantwortung, die implizit von einer herausgehobenen Machtstellung insbesondere großer Unternehmen ausgeht, mit der Leitidee des machtfreien, auf Freiwilligkeit basierenden wirtschaftlichen Beziehungsgefüges an Märkten, die eine der Grundideen bürgerlichliberaler Ordnungsvorstellung darstellt?

- Welchen Inhalt hat unternehmerische Verantwortung, wessen Interessen sind also richtungsgebend für die Unternehmenspolitik und wie sind sie im Konfliktfall zum Ausgleich zu bringen?

- Wo bleibt die Kontrolle darüber, ob das Management seine soziale Verantwortung auch tatsächlich angemessen wahrnimmt?

- Wer hat dem Management von Unternehmen die Legitimation dazu übertragen, soziale Verantwortung wahrzunehmen, und kann sie ihm bei Nichterfüllung gegebenenfalls auch wieder entziehen?

\footnotetext{
${ }^{5}$ Wohl aber nimmt er auf diverse angelsächsische Publikationen seinerseits Bezug, z.B. auf Heald (1957), Petit (1965) sowie Learned et al. (1969).
} 
Seine Antworten sind durchweg problematisierend: Es sei keine Verträglichkeit mit den Grundlagen der Marktdoktrin gegeben, so dass die These von der sozialen Verantwortlichkeit des Management eher als Abwehrargument gegen eine staatliche Intervention zur (Wieder)herstellung ausgeglichener Machtverhältnisse an den Märkten zu interpretieren sei. Der Inhalt der sozialen Verantwortung und Aussagen zu Modalitäten des Interessenausgleichs blieben ebenso vage wie die Nennung einiger ausgewählter Anspruchsgruppen willkürlich. Kontrollmechanismen blieben ebenso unerwähnt wie die Legitimationsfrage unbeantwortet. Daher sei „die Idee als eine pseudo-normative Leerformel zu qualifizieren“ (ebenda, 470), die in hohem Maße ideologisch geprägt sei, weil sie Machtstrukturen rechtfertige, Konsequenzen der Machtausübung verschleiere und angesichts vorhandener Machungleichgewichte gebotene Staatseingriffe für unnötig erkläre. Zur Bestätigung zitiert er die an Naivität kaum $\mathrm{zu}$ überbietende Aussage von Learned et al.: „Indeed, the emergence of the doctrine of social responsibility is the principal justification for leaving corporate power unchecked“(ebenda). Auf diese Argumente wird zurückzukommen sein.

Das Konzept der Sozialbilanzierung geht insofern über die bloße Proklamierung sozial verantwortlicher Unternehmensführung hinaus, als es den substantiellen Beitrag des Unternehmens zum Gemeinwohl in Form eine „Bilanz“ zu dokumentieren und öffentlich zu machen versucht. Sozialbilanzen stellen damit gleichsam ein Umsetzungsinstrument zum Nachweis der tatsächlich erfolgten sozialen Nutzenstiftung durch die Unternehmenstätigkeit dar.

Die ersten Sozialbilanzen wurden von Vorreiterunternehmen Anfang der 70er Jahre vorgelegt, von anderen aufgegriffen und weiterentwickelt, in Frankreich sogar gesetzlich vorgeschrieben, aber auch in einer breiten wissenschaftlichen Debatte kontrovers diskutiert. ${ }^{6}$ Sie können damit als Vorläufer der heutigen Umwelt- und Nachhaltigkeitsberichterstattung angesehen werden.

Es ist an dieser Stelle nicht möglich, eine ausführliche Darstellung der verschiedenen Sozialbilanzierungskonzepte, ihrer Aussagemöglichkeiten und -grenzen vorzunehmen. Stattdessen soll in aller Kürze eine Würdi-

6 Vgl. etwa Dierkes 1974 und 1984, Eichhorn 1974, Fischer-Winkelmann 1980, Vogelpoth 1980. 
gung des Phänomens und des darauf bezogenen Diskurses versucht werden.

Aus heutiger Sicht stellen Sozialbilanzen ein Phänomen dar, das durch eine weite Kluft zwischen Theorie und Praxis gekennzeichnet ist. Weitgehend unbeeinflusst von theoretisch konzeptionellen Überlegungen schritt die Unternehmenspraxis voran und legte die ersten Sozialberichte und -bilanzen vor. ${ }^{7}$ Diese Praxis wurde später in einem PraktikerArbeitskreis zusammengeführt und konzeptionell einheitlich in drei Bestandteile gegliedert (vgl. Pieroth 1978):

- die sog. Sozialrechnung, in der auf Grundlage von Zahlen aus der GuV-Rechnung Aussagen über die sozialen Wirkungen betrieblicher Prozesse in Bezug auf die wesentlichen Anspruchsgruppen (Mitarbeiter, Kapitalgeber, Staat, Öffentlichkeit, Umwelt) vorgelegt werden sollten,

- die sog. Wertschöpfungsrechnung, die - gegliedert in eine Entstehungs- und eine Verwendungsrechnung Aufschluss über Höhe, Entstehung und Verteilung der Unternehmensbeiträge zur volkswirtschaftlichen Wertschöpfung geben sollte

- und den Sozialbericht, in dem sich verbale und illustrative Erläuterungen zu den verschiedenen Posten der Sozial- und Wertschöpfungsrechnung finden ließen.

Damit stellt diese Form der Sozialbilanz8 ein Informationsinstrument dar, das weitgehend auf finanziellen Daten basiert, die der Finanzbuchhaltung, insbes. der Gewinn- und Verlustrechnung des berichtenden Unternehmens entnommen sind. Die grundlegenden Daten stellen somit Aufwendungen oder Erträge und damit den finanziellen Niederschlag der Bemühungen dar, die das Unternehmen angestrengt hat und denen interpretativ ein sozialer Nutzen zugeschrieben wird, ohne dass dieser in seinen Wirkungen gemessen und beziffert wird. Gezahlte Löhne sind dann

${ }^{7}$ So z.B. in Deutschland die Firmen Pieroth, Rank Xerox, Saarbergwerke, Steag, Shell und Rheinbraun, in der Schweiz die Migros.

${ }^{8}$ Auf andere Formen wie z.B. den Sozialbilanzentwurf des DGB und die französischen Vorschriften zur Sozialbilanz, auf die sich der DBG-Entwurf weitgehend stützt, soll an dieser Stelle nicht eingegangen werden. Vgl. aber DGB Bundesvorstand 1979 und Vogelpoth 1980. 
z.B. soziale Leistungen des Unternehmens an seine Mitarbeiter, Steuern und Abgaben sind Leistungen an den Staat, gleichgültig ob sie auf Grund vertraglicher oder gesetzlicher Verpflichtungen geleistet werden oder auf freiwilliger Basis. Kosten für eine Filteranlage sind Leistungen für die natürliche Umwelt, wie wirksam und ökologisch sinnvoll sie auch immer sein mögen.

Negative externe Effekte, wie sie seit William Kapp im Fokus der (umwelt)ökonomischen Debatte stehen (Kapp 1950), tauchen in diesen Sozialbilanzen ebenso wenig auf wie z.B. gesundheitliche Schädigungen der Mitarbeiter. Insofern waren Sozialbilanzen dieser Prägung vor allem Publikationen zur Imagepflege, Broschüren, die darstellen sollten „was ein Unternehmen für die Gesellschaft insgesamt Nutzbringendes leistet“ (Faltlhauser 1978, 163). Sie begegnen dem sozialen Druck kritischer Anspruchsgruppen, aber sie leisten nicht wirklich, was sie vorgeben zu leisten, das Ausmaß der von Unternehmen wahrgenommenen sozialen Verantwortung objektiv im Sinne von extern nachprüfbar zu dokumentieren.

Auch die Sozialbilanzierungspraxis ist mithin in großem Umfang ideologisch geprägt: die dahinter stehende Absicht, sozialer Kritik mit einer Darstellung der sozialen „Schönseite“ des Unternehmens (Budäus, 1977, 200) zu begegnen, ist allzu durchsichtig. Die Fragen, worin sich soziale Verantwortung auszudrücken hätte, wie sie sich legitimiert, wie sie kontrolliert und bei Nichtwahrnehmung sanktioniert werden soll, werden weder gestellt noch beantwortet.

Die dargestellten historischen Stationen von Versuchen, Unternehmenspolitik bzw. unternehmerisches Handeln auch als Ausdruck der Wahrnehmung sozialer Verantwortung zu charakterisieren und damit zu legitimieren, haben Mehreres gemeinsam:

- Sie entstehen durchweg zu Zeiten, in denen praktische Unternehmensführung gesellschaftlichen Moralvorstellungen zuwiderläuft und/oder verstärkter gesellschaftlicher Kritik ausgesetzt ist.

- Sie proklamieren soziale Verantwortung, ohne konkret auf deren Inhalte, Legitimation und Kontrollierbarkeit und ihre Vereinbarkeit mit vorherrschenden politischen Vorstellungen einzugehen.

- Sie verzichten vollends auf oder greifen zu unzulänglichen Instrumenten zur Umsetzung der sozialen Verantwortung im täglichen praktischen Handeln von Unternehmen. 


\section{Zum historischen Hintergrund der heutigen CSR-Debatte}

Das gilt auch für die aktuelle Debatte, wie im Folgenden nachzuweisen sein wird. Sie hat ihren sozialen Hintergrund im Zusammentreffen verschiedener gesellschaftlicher Ereignisse und Entwicklungen.

$\mathrm{Da}$ ist zum einen auf der Ebene der einzelnen Unternehmen das Obsiegen eines wirtschaftlichen Handlungsmusters zu nennen, das jenseits jeglicher Beeinträchtigungen von gesellschaftlich-moralischen Schranken das Streben nach dem eigenen finanziellen Vorteil, verbrämt als Ausdruck wirtschaftlicher Rationalität, insbesondere in managergeführten Großunternehmen zur Vorherrschaft hat gelangen lassen. Dies kann als Fleischwerdung des Homo Oeconomicus und seiner Moral missachtenden Nachfahren charakterisiert werden (Freimann 2006, 41ff.). Es äußert sich nicht nur in Fällen schierer Unersättlichkeit, was das Gewinnstreben angeht, sondern auch in zahlreichen Fällen scheinbar grenzenlosen Lohndumpings, das nicht nur in den USA immer mehr Menschen trotz Wahrnehmung eines vollen Arbeitsverhältnisses in Armut bringt. Es äuBert sich auch in Bestechung und Bestechlichkeit sowie weiteren durchaus nicht im Einklang mit gesetzlichen Vorschriften stehenden Handlungen von Unternehmensverantwortlichen.

Sein einzelwirtschaftliches Pendant findet dieses Handlungsmuster im - ebenfalls vermeintlich rationalen - Konsumhandeln vieler Menschen insbesondere in den Industrieländern. Wo Geiz geil ist, „ruiniert König Kunde sein Land"(Pötter 2006). Wer im Internet oder anderswo möglichst viele möglichst billige Produkte kauft, nimmt - ob unbedacht oder bewusst - soziale Nebenfolgen wie z.B. das Sterben des stationären wohnortnahen Handels oder unmenschlicher Arbeitsbedingungen in indischen Sweatshops in Kauf.

Auf der wirtschaftspolitischen Ebene ist nahezu weltweit ein Rückzug des Staates zu beobachten, der einerseits aus einem tatsächlichen Machtverlust der Nationalstaaten gegenüber international agierenden (Groß)unternehmen resultiert, andererseits aber auch Ausdruck des Obsiegens neoliberaler wirtschaftspolitischer Konzepte ist, wonach staatliche Regulierung mehr Fessel wirtschaftlichen Fortschritts als dessen politischer Wegbereiter und Lenker ist. ${ }^{9}$ In einer globalisierten Weltwirt-

\footnotetext{
${ }^{9} \mathrm{Ob}$ und inwieweit die aktuelle weltweite Krise diese Entwicklung aufhalten oder umkehren kann, ist aus meiner Sicht derzeit nicht auszumachen.
} 
schaft, in der immer mehr Unternehmen global beschaffen, produzieren und verkaufen, herrscht verschärfte Standortkonkurrenz und schwächt nationale Politik. Denn diese definiert sich verstärkt als Interessenvertretung der nationalen Wirtschaft und buchstabiert ihre Rolle oft als Aufforderung zur Deregulierung und Senkung standortbedingter Kosten anstatt als Aufgabe, Innovationen und Zukunftsfähigkeit zu fördern. Die Folgen: Ein Downcycling nationaler Regulierung und in dessen Folge immer weniger durchsetzungsmächtige Nationalstaaten. Auch im nationalen Rahmen wird der Staat immer mehr zum Moderator des Diskurses mehr oder weniger mächtiger Interessengruppen, was sich nicht zuletzt darin äußert, das z.B. die EU ein unverbindliches „Grünbuch“ zur sozialen Verantwortung von Unternehmen vorlegt, statt diese in gesetzlicher Form einzufordern und zu regulieren.

Demgegenüber gewinnen publizistische Medien und oft international tätige Nichtregierungsorganisationen zunehmend an Bedeutung und lenken ihre kritische Aufmerksamkeit nicht zuletzt auf skandalisierbare Phänomene im Handeln von Unternehmen. Dabei nehmen sie einerseits zumindest teilweise die Kontrollfunktionen wahr, die bisher im Aufgabenbereich des Staates lagen, indem sie das Unternehmenshandeln beobachten und kritische Befunde öffentlich machen. Andererseits verfolgen sie jedoch auch eigene Interessen wie z.B. Auflagensteigerung und Fundraising, was nicht immer der Wahrheitsfindung in Bezug auf die Objekte ihrer Aufmerksamkeit dient. Während Unternehmen also Staaten immer mehr dominieren, müssen sie gegenüber den zivilgesellschaftlichen Stakeholdern zunehmend an kooperativen oder zumindest verträglichen Beziehungen interessiert sein. CSR-Bekundungen sind Ausdruck dieses Bemühens.

Über all diesen Phänomenen erhebt sich die allgemeine Problematik, dass die westlich-industrielle Produktions- und Lebensweise zwar auf dem Wege ist, die Welt zu erobern, indem sie zum Vorbild für Schwellen- und Entwicklungsländer geworden ist, dem diese allzu oft mit dem Umweg über frühkapitalistische Technik- und Sozialstrukturen nachstreben. Andererseits ist sie unter ökologischen Gesichtspunkten nicht globalisierbar, ohne dass es mehrere Welten bräuchte. Nicht nur, aber auch Unternehmen sind auch vor diesem Hintergrund gehalten, umzusteuern und zur Sicherung ihres Ressourcenzugangs und der natürlichen Lebenszusammenhänge eine Wirtschaftsweise zu entwickeln, die nachhaltig im Sinne von sozial und ökologisch globalisierbar ist. 


\section{Zur inhaltlichen Konkretisierung von CSR}

Wie also wäre CSR vor diesem Hintergrund heute inhaltlich zu konkretisieren? Setzen wir zur Beantwortung dieser Frage noch einmal bei der CSR-Definition von Carroll an. Zunächst sei daran erinnert, dass zumindest die beiden unteren Stufen der Pyramide eher als Erfüllung existentieller Handlungsbedingungen privatwirtschaftlicher Unternehmen zu begreifen sind denn als Ausdruck sozialer Verantwortungsübernahme. Immerhin besteht bei Verstößen gegen beide Gebote die Gefahr, relativ schnell vom Markt verschwinden zu müssen, ganz gleich in welcher Weise man sonst gegenüber der Gesellschaft auftritt.

Carrolls dritte Ebene bleibt dafür, dass sie die erste und eigentlich einzige Ebene ist, die sich zentral auf das Geschäftsgebaren des Unternehmens bezieht, relativ verschwommen und interpretationsbedürftig. Buchstabiert man sie in einer Weise, dass man „be ethical“ als „do no harm“ versteht, dann wird darin zwar immer noch keine konkrete Handlungsebene angesprochen, aber die Aufforderung ablesbar, im Kerngeschäft und in den Stakeholderbeziehungen Schäden zu vermeiden. Unternehmen hätten auch die Nebenfolgen ihres Tuns und ihres Leistungsangebots mit zu bedenken und im Sinne der Schadensvermeidung zu beachten, eine durchaus weit gehende Forderung.

Ebene 4 dagegen mag als eine spezielle Form der Gewinnverwendung jedem Unternehmer als Privatperson gut zu Gesicht stehen, ist aber im Fall von managergeführten Unternehmen zumindest insofern nicht unproblematisch, weil im Namen des Unternehmens spendende Manager nicht ihr eigenes Geld, sondern das der Anteilseigner vergeben, in der Regel ohne von diesen dazu ausdrücklich legitimiert worden zu sein. Tun sie es aus Marketinggründen als Bestandteil der Unternehmenswerbung, dann ist es gerade nicht Ausdruck sozialer Verantwortung, sondern wie Ebene 1 und 2 auch business as usual.

So kommt in dieser Ausprägung CSR überwiegend daher als mehr oder weniger zwangsweise Befolgung existentieller Handlungsbedingungen oder als Gewinnverwendung durch den Unternehmer als Privatperson oder dazu nicht legitimierte Manager. Dort wo sie das Kerngeschäft betrifft, klingt sie anspruchsvoll, bleibt aber unbestimmt. Eine Inhaltsbestimmung dessen, worauf denn CSR auf der Ebene ethisch geprägter Unternehmensführung abheben könne oder müsse, wird ebenso wenig geführt wie nach der Legitimation, institutioneller Kontrolle und den Me- 
chanismen des Verantwortungsentzugs bei Nichtwahrnehmung gefragt wird. Das so transportierte CSR-Verständnis kann mithin nur als erneuter ideologischer Legitimationsversuch für in Kritik geratene, weil selbst gegen Rechtsnormen und gesellschaftliche Wertvorstellungen verstoßende Unternehmensführung charakterisiert werden.

Im europäischen bzw. deutschen CSR-Diskurs kommt eine etwas klarere Trennung zwischen Corporate Citizenship (CC) und CSR zum Tragen, bei der CC zumeist weitgehend mit philanthropischem Engagement identifiziert wird, allerdings ohne genauer zu prüfen, ob und in welchem Maße sowie unter Bezug auf welches Verständnis von Bürgertum die Rolle eines Unternehmens als gewinnorientierter Spieler im Wettbewerbszusammenhang vereinbar ist mit den Anforderungen an bürgerschaftliches Engagement (vgl. aber Schrader 2003). CSR dagegen wird zumeist enger gefasst und stärker auf Aktivitäten im Zusammenhang mit dem Kerngeschäft bezogen. Inhaltlich finden sich in größerem Umfang Bemühungen, CSR als praktisch-instrumentellen Ausdruck einer nachhaltigen Unternehmensführung zu verstehen (Loew et al. 2004).

Dabei wird allerdings, wie oben bereits angemerkt, Nachhaltigkeit nicht nur in der EU-Definition sondern auch in weiten Teilen der Unternehmenspraxis mit dem sog. Drei-Säulen-Modell operationalisiert, nach dem die „Dimensionen Ökologie, Ökonomie und Soziales in ein (dynamisches) Gleichgewicht zu bringen sind“ (Majer 2000, 383). Nachhaltige Entwicklung wird so nicht mehr von der Leitidee einer intra- und intergenerationalen Gerechtigkeit her gedacht, nach der alle aktuell Lebenden und die zukünftigen Generationen das prinzipiell gleiche Recht auf angemessene Lebenschancen haben, also niemand berechtigt ist, ein Lebensmodell zu praktizieren, das nicht globalisierbar, d.h. von jedermann/frau lebbar ist. Stattdessen werden soziale und wirtschaftliche Belange mit ökologischen Belangen (denn die verfügbaren Naturvorräte und Aufnahmekapazitäten der Natur für die Abfälle menschlichen Wirtschaftens sind - zumindest für die entwickelten Länder - die eigentlichen Engpassfaktoren für die globale Umsetzung nachhaltiger Entwicklung im oben genannten Sinne) auf eine Stufe gestellt. Paech bemerkt hierzu kritisch: „Nachhaltig kann also nur sein, was im Schnittmengenbereich der drei Säulen liegt“" (Paech 2005, 93).

„Win-win-win“ heißt die Devise. Das heißt nicht nur, dass die Bewahrung der natürlichen Lebensgrundlagen nur dann nachhaltig im Sinne des Drei-Säulen-Modells ist, wenn sie zugleich ökonomischen und sozialen 
Belangen förderlich ist, also der Gewinnerzielung dient und auf soziale Akzeptanz stößt. Es heißt damit, dass die eigentlich bedeutsamen Konfliktfelder zwischen wirtschaftlichen und sozialen Interessen einzelner Gruppen und/oder Unternehmen aus dem Blick geraten, jedenfalls aber auf Grundlage des Modells nicht bearbeitet werden können.

Für Unternehmen (wie natürlich auch für andere gesellschaftliche Akteure) wird damit das Tor zur Beliebigkeit groß aufgemacht. Letztlich ist alles Handeln, das sich irgendwie als sozialen, ökonomischen und ökologischen Belangen förderlich interpretieren lässt, ein Schritt auf dem Weg zur Nachhaltigkeit. Und umgekehrt kann niemand mehr Effizienzsteigerungen (z.B. geringere $\mathrm{CO}_{2}$-Emissionen von Autos pro $100 \mathrm{~km}$ ) deshalb als nicht nachhaltig kritisieren, weil sie durch Flottenwachstum und steigende Fahrleistungen überkompensiert werden, denn diese sind ja sozial und ökonomisch erwünscht.

Wollte man CSR in dem Sinne inhaltlich füllen, dass sich in ihr Nachhaltige Unternehmensführung manifestieren soll/kann, dann scheint mir der Bezug auf Win-Win-Win kontraproduktiv. Denn damit wird alles und nichts, was ein Unternehmen sozial und ökologisch bewirkt oder beeinflusst, als Beitrag zu einer nachhaltigen Entwicklung interpretierbar. Substantiell prüfbare Maßstäbe fehlen völlig. Zum Umgang mit Konfliktsituationen gibt es keine Hinweise. Auch in dieser Lesart erweist sich CSR damit als inhaltlich unbestimmt und ideologieverdächtig.

Von zentraler Bedeutung ist jedoch die Frage, ob und in welchem Maße auf gesellschaftliche Nutzenstiftung oder Schadensvermeidung zielende Aktivitäten von Unternehme(r)n Bezüge zum unternehmerischen Kerngeschäft aufweisen.

Wer sein Geld auch dazu verwendet, Anderen und der Natur Gutes zu tun, verdient Anerkennung. Wenn jedoch Volkswagen die Kasseler Documenta fördert oder Otto Geld für soziale Einrichtungen spendet, dann leisten die Unternehmen soziale Beiträge aus erzielten Gewinnen (auch wenn Spenden steuerlich als Betriebsausgaben und damit gewinnmindernd verbucht werden können). ${ }^{10}$ Wenn gar Krombacher von dem

\footnotetext{
${ }^{10}$ Gesellschaftlich problematisch ist dann allenfalls, dass derartige soziale Fördermaßnahmen keinerlei demokratische Legitimation haben und damit dort, wo sie staatliche Maßnahmen ersetzen oder ergänzen, den privaten Förderern das Entscheidungsrecht überlassen wird, was sie für förderungswürdig halten, anstatt es bei den dazu durch Wahlen legitimierten Instanzen zu belassen und diese finanziell besser auszustatten.
} 
Erlös jedes Kastens Bier ein paar Cent zur Erhaltung des tropischen Regenwaldes spendet oder UHU die Wiederansiedlung des gleichnamigen Großvogels in Deutschland unterstützt, dann sind diese Spenden in Wirklichkeit nichts als Ausgaben für Unternehmens- oder Produktwerbung. Sozial verantwortbare Unternehmenspolitik hieße hier z.B. auf gesüßte Biermixgetränke zu verzichten, weil durch sie der Alkoholmissbrauch bei Jugendlichen gefördert wird oder den Klebstoff so zu verändern, dass suchtförderndes Schnüffeln nicht mehr möglich ist.

Substantiell sozial verantwortliche Unternehmensführung also ist etwas Anderes. Sie beweist sich im Prozess der Gewinnerzielung. Sozial verantwortlich handeln Unternehmen, die ihren MitarbeiterInnen auskömmliche Löhne zahlen, gesundheitlich beeinträchtigende Arbeitsbedingungen beseitigen oder gar nicht erst in Kauf nehmen, von Produktionsprozessen oder Produkten ausgehende Umweltrisiken vermeiden oder zumindest weitestmöglich verringern, ihren Kunden qualitativ hochwertige Produkte anbieten ..., kurzum die in ihrem Geschäftsgebaren Moral über Geschäft stellen. Soziale Verantwortung zu übernehmen heißt also, legitimes Gewinnstreben einbetten in Normen, die gesellschaftlich akzeptiert und ethisch begründet sind. ${ }^{11}$ Wenn eine solche Norm diejenige der Nachhaltigkeit im Sinne des Übertragbarkeitskriteriums ist, dann bin ich geneigt, so verstandene CSR als unternehmenspolitischen Beitrag zur Nachhaltigen Entwicklung zu bezeichnen.

\section{Zur institutionellen Verankerung von CSR}

Ist damit die bereits von Horst Steinmann in Bezug auf das Davoser Manifest festgestellte inhaltliche Unbestimmtheit von CSR beseitigt, dann sind nun noch seine Fragen nach der institutionellen Legitimität und Kontrolle der sozialen Verantwortungsübernahme von Unternehmen aufzugreifen. ${ }^{12}$ Wer oder was legitimiert Unternehmensleitungen zur Wahr-

\footnotetext{
${ }^{11}$ Etwa nach dem vom Thomas Mann kolportierten Grundsatz der Lübecker Kaufmannsfamilie Buddenbrook, der da lautet: "Mein Sohn, sey mit Lust bey den Geschäften am Tage, aber mache nur solche, daß wir bey Nacht ruhig schlafen können.“ (Buddenbrooks, 2. Teil, 1. Kapitel).

12 Auf den von Steinmann ebenfalls problematisierten Zusammenhang zwischen soziale Verantwortung begründender Macht einerseits und marktwirtschaftlich-
} 
nehmung sozialer Verantwortung, mittels welchen Mechanismus' kann diese Legitimation bei Nichterfüllung entzogen werden und wie kann Kontrolle ausgestaltet werden darüber, ob, mit welchen Inhalten und mit welchen Erfolgen die Verantwortungsübernahme praktiziert wird?

Nach meiner Kenntnis werden diese Fragen in der aktuellen CSRDebatte nur am Rande und vielfach unzureichend thematisiert oder beantwortet. Es heißt nämlich, die Legitimation sei gleichsam implizit dadurch gegeben, dass nur Unternehmen, die CSR übernehmen, die sog. „licence to operate“ erhielten, die ihnen andernfalls entzogen würde.

Hier ist wohl eher der Wunsch Vater des Gedankens. Wer entzöge z.B. der Atomindustrie die Geschäftsgrundlage, die seit Jahrzehnten ohne Entsorgungsnachweis risikoreiche Atomreaktoren betreibt, die eine Mehrheit der Bevölkerung ablehnt? Selbst Unternehmen, die nachweislich in gravierender Weise die Gesundheit ihrer Mitarbeiter und Anlieger schädigen, stoßen zwar auf soziale Kritik, finden aber selbst im Konfliktfall nicht selten milde Richter. Und Bankinstitute, deren Handeln die bisher größte Weltwirtschaftskrise zumindest billigend in Kauf genommen hat, werden zwar in den Medien und von der Politik kritisiert, aber mit dem Hinweis auf unüberschaubare „Systemrisiken“ durch massive staatliche Hilfen vor dem Konkurs gerettet. Ein wirklicher Entzug der sozialen Akzeptanz ist mir zumindest noch nicht begegnet. Und mit sozialer Kritik gehen Unternehmer und Manager nicht selten so um, dass sie ihr die fachliche Kompetenz abstreiten (vgl. z.B. Heine/Mautz 1995), sie also kaum je als Anstoß zum Nachdenken aufgreifen.

Institutionelle Legitimierungs- und Kontrollstrukturen sind also unerlässlich. Beginnen wir mit den Kontrollmöglichkeiten. Deren Ausgestaltung kann sowohl an historische Erfahrungen im Bereich der finanzökonomischen Kontrolle auf dem Weg der verbindlichen Informationsvorschriften für die bilanzielle Rechnungslegung und Geschäftsberichterstattung anknüpfen als auch an entsprechende Erfahrungen der Sozialbilanzierung.

Hier ist die Entwicklung der CSR- oder Nachhaltigkeitsberichterstattung auf einem guten Wege. Nachdem sich der von Frankreich eingeschlagene Weg einer gesetzlichen Verpflichtung zur Vorlage einer inhaltlich präzis definierten Sozialbilanz international nicht hat durchsetzen 
können (vgl. Dierkes 1984, 1230/31), und frühe Vorschläge zur Standardisierung der betrieblichen Ökobilanzierung keine Resonanz fanden (Freimann 1990), ist seit Beginn der 90er Jahre eine fast schon stürmische Entwicklung auf dem Feld der freiwilligen Umwelt- und Nachhaltigkeitsberichterstattung zu beobachten (Kahlenborn et al. 2007). Zunächst wurde vereinzelte Umweltberichte von Unternehmen vorgelegt. Seit 1996 schnellte die Zahl der einschlägigen Berichte auf viele Tausend hoch, befördert durch die europäische EMAS-Verordnung, die die Veröffentlichung einer Umwelterklärung den Teilnehmern am System zwingend vorschreibt. Sie stagnierte dann aber mit den EMASTeilnehmerzahlen, auch weil das weltweit gültige und erheblich erfolgreichere ISO 14001-System keine entsprechenden Publikationspflichten enthält.

Inzwischen aber legen nicht nur viele (große) Unternehmen freiwillige Nachhaltigkeits- oder CSR-Berichte vor, sondern es hat sich mit den GRI-Richtlinien ein weltweiter Standard zur entsprechenden Berichterstattung etabliert, an dem sich immer mehr berichtende Unternehmen orientieren (www.globalreporting.org). Die Richtlinien enthalten Vorgaben zu den wichtigsten Inhalten eines Berichts, die auf freiwilliger Basis übernommen werden können, aber nur im Bericht selbst als Bezugsgrundlage erwähnt werden dürfen, wenn sie zumindest in gewissem Umfang verbindlich eingehalten wurden. ${ }^{13}$

Kritisch anzumerken bleibt jedoch, dass die GRI Guidelines nur einen weiten Rahmen darstellen, der inhaltlich nicht vollständig ausgefüllt werden muss und daher noch immer dazu führt, dass die meisten Nachhaltigkeits- oder CSR-Berichte wie seinerzeit die Sozialbilanzen als PRInstrumente aufgezogen werden, die die Schönseite des unternehmerischen Engagements darstellen und fortbestehende Probleme und Schwächen nicht oder allenfalls am Rande erwähnen. Insofern besteht offensichtlich auch die Differenz zwischen Theorie und Praxis fort, die Fischer-Winkelmann schon 1980 für die Sozialbilanz-Diskussion konstatiert hat (Fischer-Winkelmann 1980, 6ff.) und die darin besteht, dass Wissenschaftler mit dem sozial-ökologischen Reporting von Unterneh-

\footnotetext{
${ }^{13}$ In jüngster Zeit mehren sich jedoch kritische Stimmen, die die GRI-Guidelines mit ihrer Fixierung auf westlich-industriell relevante Berichtsinhalte als nicht verallgemeinerbar insbesondere für Schwellen- und Entwicklungsländer mit abweichenden Kulturen und sozialen Problemen beurteilen. (z.B. Brown et al. 2009).
} 
men Ansprüche einer umfassenden Rechenschaftslegung verbinden, die so von der Unternehmenspraxis nicht geteilt werden und die die Nationalstaaten in der globalisierten Welt ja nicht einmal im Bereich der finanzwirtschaftlichen Rechnungslegung verlässlich einzufordern in der Lage sind.

Lediglich die kritische Öffentlichkeit und durchsetzungsmächtige marktliche Anspruchsgruppen könnten hier Ansprüche geltend machen und durchsetzen. Tatsächlich gibt es Entwicklungen in dieser Richtung: einschlägige Rankings in der Wirtschaftspresse ${ }^{14}$ sowie das verstärkte Interesse, das nachhaltigkeitsorientierte Rating-Agenturen diesen Berichten entgegenbringen, um verlässliche Grundlagen für ihre Beurteilung des nachhaltigkeitsbezogenen Engagements der Unternehmen zu bekommen, die in einschlägigen Börsenindices geführt oder in einschlägige Fonds aufgenommen werden wollen. So sind es der Kapitalmarkt und die interessierte Öffentlichkeit, die hier die Lücke zu füllen versuchen, die die zunehmend schwächere staatliche Regulierung hinterlässt.

Der Kapitalmarkt hat über das Interesse einer wachsenden Zahl von Investoren an nachhaltigen Geldanlagen einen wichtigen Impuls gesetzt, um auf dem Gebiet der CSR zumindest halbwegs solide Kontrollmöglichkeiten zu etablieren. Allerdings scheint das Öko-Rating zumeist mit relativ oberflächlichen Informationen zufrieden zu sein. Den in diese Richtung berichtenden Unternehmen geht es daher oft nur um die Abwehr des Risikos erhöhter Kapitalkosten. Proaktive Nachhaltigkeitsorientierung sieht anders aus. Zudem haben die Rating-Agenturen in der aktuellen Finanzmarktkrise viel Renommee verspielt, indem sie die finanziellen Risiken vieler Investments notorisch unterschätzt und so einen erheblichen Beitrag zur Verschärfung der Krise geleistet haben, anstatt vor den sich abzeichnenden Entwicklungen frühzeitig zu warnen.

Kritischer und mit mehr ökologischem und sozialem Sachverstand betrachten NGOs und deren Repräsentanten die CSR- und Nachhaltigkeitsberichte von Unternehmen. Sie legen die Latte ihrer Ansprüche deutlich höher und verfügen mit dem Instrument der Skandalisierung auch über ein nicht zu unterschätzendes Durchsetzungspotential. Was sie relativ wenig einsetzen, ist das Setzen von positiven Incentives, die engagierte Unternehmen belohnen und so das Engagement und nicht nur die Risikoabwehr befördern. Eine Belohnung etwa in Form einer Auszeichnung

${ }^{14}$ In Deutschland vor allem durch die Zeitschrift Capital und das Berliner IÖW. 
„,nachhaltiges Unternehmen“, verliehen durch ein entsprechendes Gremium von NGO-Vertretern, könnte hier viel bewirken.

Solange all dies jedoch ohne jede verpflichtende Regulierung abläuft, weil Nationalstaaten zu wenig Macht haben oder sich programmatisch zurückziehen und harte supranationale Regulierung fehlt, wird abzuwarten sein, ob es eine Modeerscheinung bleibt oder ob der Prozess der weichen zivilgesellschaftlichen Selbstregulierung dazu führen wird, dass die Kontrollmöglichkeiten im Sinne der Bereitstellung valider und vergleichbarer Informationen sich weiter verfestigen und verbessern. Nicht zuletzt wird diese Frage an den Märkten selbst entschieden, die einschlägige Informationen nachfragen und ihre Sanktionsmöglichkeiten einsetzen müssen, um die Entwicklung zu befördern.

Institutionelle Legitimation, Kontrolle und (positive wie negative) Sanktionierung kann jedoch meines Erachtens nicht über bloße Informationsbereitstellung erfolgen. Denn auch gut informierte externe Stakeholder haben nur sehr begrenzte Einwirkungsmöglichkeiten und Einwirkungsinteressen in Bezug auf Unternehmen (vgl. Freimann 2000). Nur wenn man ihre Repräsentanten zu Insidern macht und ihre Einwirkungsmöglichkeiten auf das Management institutionell verankert, können Legitimationsgrundlagen errichtet werden, die die Möglichkeit für engagierte und dauerhaft wirksame soziale Verantwortungsübernahme von Unternehmen schaffen.

Auch hier gibt es Vorbilder und Anknüpfungsmöglichkeiten. Sie liegen insbesondere in Gestalt der deutschen Mitbestimmung sowie in Gestalt der diversen Stakeholderdialoge vor, die von Unternehmen zu konkreten Problemsituationen eingerichtet und durchgeführt wurden.

Die deutsche Montanmitbestimmung wurde in den 50er Jahren nicht in erster Linie deshalb gesetzlich verankert, um den Arbeitnehmern Einflussmöglichkeiten auf die Gestaltung ihrer Arbeitsplätze zu geben, sondern um zu vermeiden, dass sich deutsche Unternehmen noch einmal als politische Steigbügelhalter der Nazis oder ähnlicher politischer Kräfte betätigen. Sie sollte sicherstellen, dass mitbestimmte Unternehmen im demokratischen Sinne soziale Verantwortung übernehmen. Im Verlauf des Niedergangs der deutschen Montanindustrie hat sie sich zudem als Garant des sozialen Friedens bewährt. ${ }^{15}$ Das Manko der AR-

${ }^{15}$ Dass die großen deutschen Unternehmensverbände 1976 eine Verfassungsklage die Übertragung der Aufsichtsrats-Mitbestimmung auf die Gesamtwirtschaft ver- 
Mitbestimmung ist heute ihr weitgehend basisferner Charakter, der z.B. AN-Vertreter der IG Metall dazu gebracht hat, im Vodaphone-Fall selbst der Gewährung gerichtsnotorisch überzogener Tantiemen und Abfindungen willfähriger Manager ihre Zustimmung nicht zu verweigern. Zudem scheint es im internationalen Bereich kaum eine Chance zu geben, die institutionelle Mitbestimmung der Arbeitnehmer geographisch auszuweiten oder zu pluralisieren, indem weitere Anspruchsgruppen einbezogen werden.

Stakeholderdialoge sind ein verhältnismäßig neues Instrument des Stakeholdermanagements. Institutionalisiert finden sie sich in einigen deutschen Umweltgesetzen, die die Genehmigung bestimmter Anlagen an die Öffentlichkeitsbeteiligung koppeln. Auf freiwilliger Basis gibt es sie in vielfältiger Ausprägung, nicht selten auf spezielle Projekte bezogen, für die sich Unternehmen öffentliche Akzeptanz sichern wollen. Ihr Manko ist die Beliebigkeit im Umgang mit den erzielten Konsensen. So zeigt sich am Beispiel des Bürgerbeteiligungsverfahrens für den Ausbau des Flughafens Frankfurt/Main, dass trotz im Dialog erzielter Einigkeit über Nachtflugverbote diese nun doch gerichtlich durchgesetzt werden müssen, weil sich das Management dem Kompromiss nicht verpflichtet fühlt.

Ob es also in der Unternehmensordnung fest verankerte institutionalisierte Formen der demokratischen Legitimierung und Kontrolle von Unternehmenshandeln im Allgemeinen und CSR im Besonderen sind oder fallweise projektbezogene Formen, beide Spielarten bedürfen fester Regularien und einer demokratischen Rückbindung zu denen, die in ihnen von Repräsentanten vertreten werden, damit sie ihre Wirksamkeit entfalten und nicht zu Schein-Legitimationsveranstaltungen verkommen. $\mathrm{Zu}$ diesen gehört eine angemessene Informationsgrundlage ebenso wie die Verbindlichkeit der erzielten Ergebnisse und die Möglichkeit, im Falle von Nichterfüllung die Akteure zu sanktionieren.

Natürlich ist zu fragen, wie eine derart um pluralistisch-demokratische Elemente ergänzte Unternehmensordnung auf den Weg gebracht werden könnte angesichts der Schwäche der eigentlich dazu legitimierten staatlichen Institutionen. Diese Frage möchte ich als Betriebswirt zuständigkeitshalber an die Politikwissenschaft weiterreichen. Als interessierter 
Laie auf diesem Gebiet meine ich aber mich zu erinnern, dass zahlreiche Politiker im Angesicht der aktuellen Finanzkrise nach einer wirksamen internationalen Regulierung gerufen haben. Derzeit scheint es so, als wären diese zum Teil vollmundigen Forderungen bereits wieder in Vergessenheit geraten.

\section{Literatur}

Caroll, A.B.: The Pyramid of Corporate Social Responsibility: Toward the Moral Management of Organizational Stakeholders, in: Business Horizons, July/August 1991, 39-48

Brown, H.S. et al.: Building institutions based on information disclosure: lessons from GRI's sustainability reporting, in: Journal of cleaner production 17 (6) 2009, 571-580

DGB Bundesvorstand: Beschluss zu unternehmerischen Sozialbilanzen vom 12.6. 1979, Düsseldorf 1979

Dierkes, M.: Die Sozialbilanz - Ein gesellschaftsbezogenes Informations- und Rechnungssystem, Frankfurt 1974

Dierkes, M.: Gesellschaftsbezogene Berichterstattung - Was lehren uns die Experimente der letzten 10 Jahre? In: ZfB 54. Jg. 1984, 1221-1235

Dyllick, Th./Hockerts, K.: Beyond the business case for corporate sustainability, Business Strategy and the Environment, 11 (2) 2002, 130-141

Eichhorn, P.: Gesellschaftsbezogene Unternehmensrechnung, Göttingen 1974

EU-Kommission: Grünbuch. Europäische Rahmenbedingungen für die soziale Verantwortung der Unternehmen, KOM (2001) 366 endgültig, Brüssel 2001

Fischer-Winkelmann, W.: Gesellschaftsorientierte Unternehmensrechnung, München 1980

Freimann, J.: Plädoyer für die Normierung von betrieblichen Öko-Bilanzen, in: ders. (Hrsg.): Ökologischer Herausforderung der Betriebswirtschaftslehre, Wiesbaden 1990, 177-195 
Freimann, J.: Der überforderte Stakeholder - Was dürfen, können und wollen die Anspruchsgruppen der Unternehmenspolitik wissen? In: Ökologisches Wirtschaften, Heft 2 2000, 17-19

Freimann, J.: Nachhaltig wirtschaften! Wider die Orientierung des praktichen wirtschaftlichen Handelns am Vorbild der Homunkuli, in: Göllinger, Th. (Hg.) Bausteine einer nachhaltigkeitsorientierten Betriebswirtschaftslehre, Festschrift für Eberhard Seidel, Marburg 2006, 35-56282

Garriga, E./ Melé, D.: Corporate social responsibility theories: Mapping the territory, Journals of Business Ethics, 53 (1-2) 2004, 51-71

Habisch, A. / Wegener, M.: Overcoming the Heritage of Corporatism, in: Habisch, A. et al. (Hrsg.) Corporate Social Responsibility Across Europe, Berlin u.a.O. 2005, 111-123

Heald, M.: Management's Responsibility to Society: The Growth of an Idea, in: The Business History Review 31 1957, 375 ff.

Heine, H./ Mautz, R. Die Öffnung der Wagenburg? - Antworten von Chemiemanagern auf ökologische Kritik, Frankfurt/ New York 1995

Kahlenborn, W. et al.: Nachhaltigkeitsberichterstattung von Unternehmen, Stastus Quo Report Deutschland 2007, BMU (www.bmu.de/files/ pdfs/allgemein/application/pdf/nachhaltigkeit_statusbericht2007.pdf

Kapp, W.: Social Costs of Private Enterprise, Cambrigde Mass. 1950

Learned, E.P. et al.: Business Policy, Text and Cases, Homewood/Ill. 1969, $485 \mathrm{ff}$.

Löffelholz, J.: Geschichte der Betriebswirtschaft und der Betriebswirtschaftslehre, Stuttgart 1935

Loew, Th. Et al. (2004): Bedeutung der internationalen CSR-Diskussion für Nachhaltigkeit und die sich daraus ergebenden Anforderungen an Unternehmen mit Fokus Berichterstattung, Endbericht an das BMU, Berlin/Münster

Majer, H.: Das Nachhaltige Unternehmen - Versuch einer Begriffsbestimmung, in: Beschorner, Th./ Pfriem, R. (Hrsg.): Evolutorische Ökonomik und Theorie der Unternehmung, Marburg, 2000, 377-417

Matten, D. / Moon, J.: „Implicit“ and „Explicit“ CSR: A conceptual framework for a comparative understanding of Corporate Social Responsibility, in: Academy of Management Review, 33(2) 2008, 404-424

Paech, N.: Nachhaltiges Wirtschaften jenseits von Innovationsorientierung und Wachstum, Marburg 2005

Petit, Th.A.: Managerial Ethics and Social Responsibility, The Doctrine of Socially Responsible Management, in: Arizona Review 14, Nr. 2 1965, 1 ff.

Pieroth, E. (Hrsg.): Sozialbilanzen in der Bundesrepublik Deutschland, Wien/ Düsseldorf 1978 
Schrader, U.: Corporate Citizenship - Die Unternehmung als guter Bürger? Berlin 2003

Steinmann, H.: Zur Lehre von der „Gesellschaftlichen Verantwortung der Unternehmensführung“ - Zugleich eine Kritik des Davoser Manifests - in: WiSt, Heft 10, Oktober 1973, S. 467-473

Vogelpoth, N.: Die französische Sozialbilanz, Frankfurt 1980

Weyermann, M./ Schönitz, H.: Grundlegung und Systematik einer wissenschaftlichen Privatwirtschaftslehre und ihre Pflege an Universitäten und Fachhochschulen, Karlsruhe 1912 